\title{
Klassische OP- und Repositionstechniken, die man kennen sollte
}

Thomas Freude

\section{Vorspann}

Die Frage nach der „optimalen“ Frakturversorgung ist auch heutzutage ein zentrales Thema der täglichen Praxis eines/-r Traumatologen/-in und der unfallchirurgischen Forschung. Zu den „Standardverfahren“ werden Nagelund Plattenosteosynthesen gezählt, die den anatomischen Gegebenheiten angepasst sind, die möglichst unter Schonung der Weichteile eingebracht werden können und über ein spezielles Design der notwendigen Stabilität Rechnung tragen. Wir sprechen von sog. biologischen Osteosynthesen [1]. Dieser Begriff, der sich in den letzten Jahren zunehmend etablierte, beschreibt den Prozess weg von einer offenen, direkten Reposition der Frakturfragmente - mit der Notwendigkeit von großen Zugängen und Ablösung der umgebenden Weichteile - hin zur indirekten Reposition mit minimalinvasiver, frakturferner Einbringung des Osteosynthesematerials.

Trotz der rasanten Entwicklung neuer Implantate ist nicht immer ein optimales Ausheilungsergebnis zu erzielen. Es bleibt die Frage: Warum kam es in diesem oder jenem Fall zu einem diskutablen Repositionsergebnis? Was führte zum sekundären Repositionsverlust oder zu einem Implantatversagen oder zu einer ausbleibenden Frakturheilung?

Bei der Analyse zeigt sich als ein wesentlicher Faktor die gewählte OP- und Repositionstechnik.

\section{Repositionstechniken}

In den vergangenen Jahrzehnten wurden verschiedene Hilfsmittel entwickelt, um die Reposition von Frakturen, insbesondere von langen Röhrenknochen, zu erleichtern.

\section{Fixateur externe}

Das Arbeitspferd in der Unfallchirurgie ist der Fixateur externe. Dabei kann der modulare Fixateur externe sowohl in der Akutbehandlung als auch als statisches Hilfsmittel bei der endgültigen Osteosynthese bis hin zur Ausbehandlung von Frakturen bei Infekten oder schwierigen Weichteilverhältnissen verwendet werden.
Bei der Anlage des Fixateurs externe sind einige wesentliche Dinge zu beachten, die häufig vernachlässigt werden. Der 1. Schritt vor Setzen der Schanz-Schrauben sollte sein, sich Gedanken zu machen, wo diese idealerweise platziert werden können. Dabei sind neben den Loci tipici, die dem AO-Manual oder der Fixateur-externe-Schautafel zu entnehmen sind, die „safe zones“ zu bedenken, die eine Verletzung von vulnerablen Strukturen weitgehend vermeiden. Insbesondere ist immer wieder eine Verletzung des N. radialis zu beobachten, da beim Setzen des distalen Pins im Humerusschaft die Lage des N. radialis nicht respektiert wird; ein iatrogener Schaden, der bei korrekter Pin-Platzierung leicht vermieden werden kann. Des Weiteren kann durch die vorauseilende Planung, unter Einbezug der eventuellen endgültigen Osteosynthese, eine deutliche Erleichterung für den Operateur erfolgen. Beispielhaft sei hier die Anlage der Schanz-Schrauben bei distalen Femurschaftfrakturen genannt; wenn der Erstoperateur die Schanz-Schrauben nicht streng von lateral im Schaft setzt, sondern einen Winkel von $30-45^{\circ}$ von ventral wählt, lässt sich der Fixateur auch bei der Ausversorgung mittels Platte beibehalten und ist ein hervorragender statischer Assistent. Die Zugangsinzisionen zum Bohren und Setzen der Schanz-Schrauben sollten großzügig gewählt werden, da 1. durch den Einsatz von Langenbeck-Haken eine Schonung z.B. der nervalen Strukturen gewährleistet wird und 2. durch das Repositionsmanöver die Haut verzogen wird sowie bei Nichtbeachtung Druckläsionen an den Pin-Stellen erleidet. Der Aufbau des Fixateurs sollte immer in modularer 3Rohr-Technik erfolgen, um in allen Ebenen eine freie Beweglichkeit zu erlauben, bis das Repositionsergebnis zufriedenstellend ist. Dabei werden im 1. operativen Schritt die beiden Schanz-Schrauben proximal und distal der Fraktur mittels einer kurzen Fixateur-Stange verbunden. Diese „Handgriffe“ dienen der Manipulation der Schaftanteile zueinander und erleichtern diese auch. Im 2. operativen Schritt wird über eine zwischengeschaltete 3. Fixateur-Stange durch eine Rohr-zu-Rohr-Verbindung die reponierte Fraktur fixiert. Diese modulare 3-Rohr-Technik erleichtert den Aufbau des Fixateurs deutlich und führt durch die Repositionsfreiheit in 3 Ebenen ohne Verspannungen zu besseren Frakturstellungen. Darüber hinaus ist bei begleitendem Weichteilschaden die Platzierung der Schanz-Schrauben deutlich erleichtert ( $\bullet$ Abb. 1). 


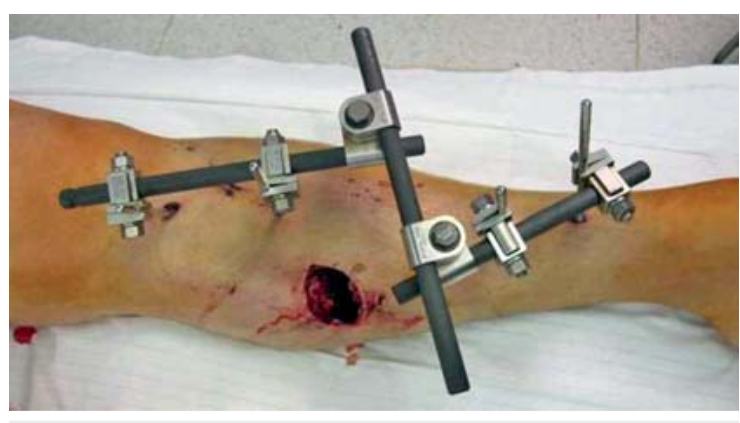

- Abb. 1 Fixateur externe in modularer 3-Rohr-Technik. Nach der Reposition und passagerer Stabilisierung muss noch ein Neutralisationsstab ergänzt werden.

Merke

Zu beachten:

- modulare 3-Rohr-Technik zur verbesserten Repositionskontrolle

- Bohren der Insertionslöcher immer mit Spülung zur Kühlung

- bikortikales Setzen der Schanz-Schrauben

- Eindrehen der Schanz-Schrauben per Hand

- übersehene Ausläufer der Fraktur zur SchanzSchraube

\section{Rahmenfixateur}

Bei der Versorgung von Unterschenkelfrakturen kann als 1. Operationsschritt ein Rahmenfixateur angebaut werden. Dabei wird im Bereich des Tibiakopfes ein Pin parallel zur Tibiaplateauebene im ventralen Drittel eingebracht. Eine Blockierung des Eintrittsweges des Tibianagels sollte vermieden werden. Und ein 2. Pin wird durch den Kalkaneus eingebracht, vergleichbar der Platzierung des Kalkaneus-Pins beim Steigbügelfixateur. Durch diese Rahmenkonstruktion ist primär die Fraktur annähernd reponiert und der Nagel über die Fraktur leichter einzubringen ( $\triangleright$ Abb. 2). Ein weiterer Vorteil ergibt sich durch die Achsstabilität, sodass beim Einschlagen des Nagels die Weichteile nicht zusätzlich kompromittiert werden, da der Rahmen das Widerlager beim Vortreiben des Nagels bildet; somit werden die Weichteile nicht gestaucht.

\section{AO-Distraktor}

Leider ist der altgediente AO-Distraktor in vielen Abteilungen in Vergessenheit geraten und fristet ein Dasein in Versorgungslagern. Dabei ist er von unschätzbarem Wert bei der Versorgung von Femurschaftbrüchen. Bedingt durch den Verstellmechanismus in allen 3 Achsen lassen sich die muskulären Zugkräfte am Oberschenkel neutralisieren und die anatomischen Verhältnisse in Länge, Rotation und Achse vor der intramedullären Versorgung herstellen. Gerade in Zeiten der verknappten Personalressourcen ist der Distraktor eine perfekte Unterstützung, die eine schrittweise Reposition ermöglicht und in der einmal gewählten Stellung verharrt. In $>\mathbf{A b b} \mathbf{3}$ ist der

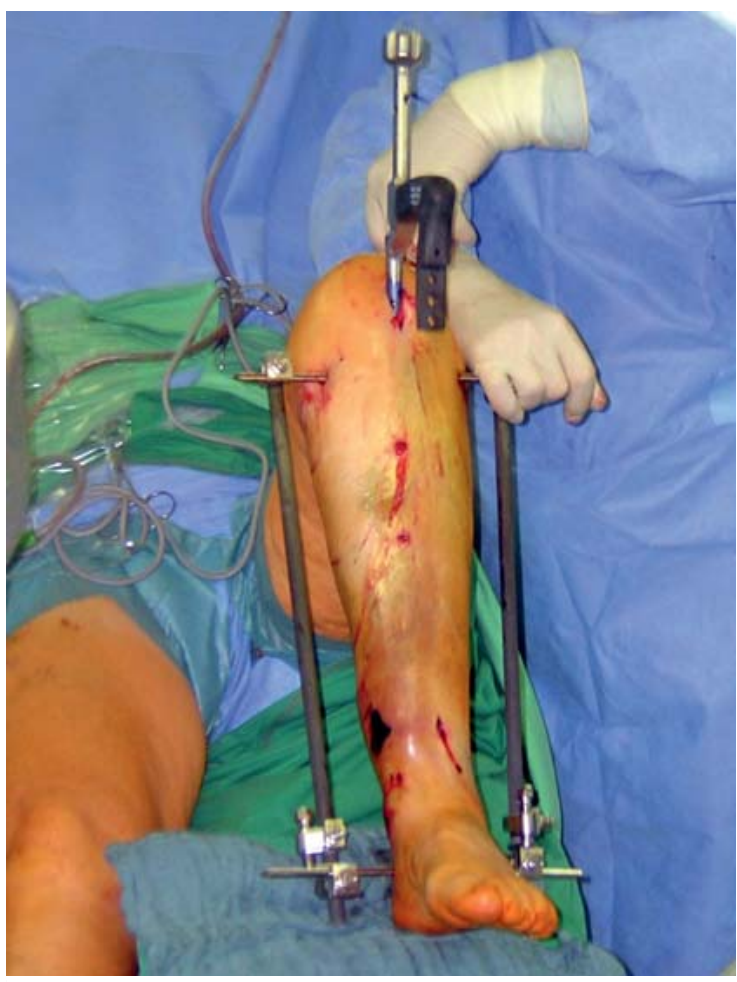

- Abb. 2 Rahmenfixateur als 1. Schritt der intramedullären Nagelosteosynthese der Tibia.

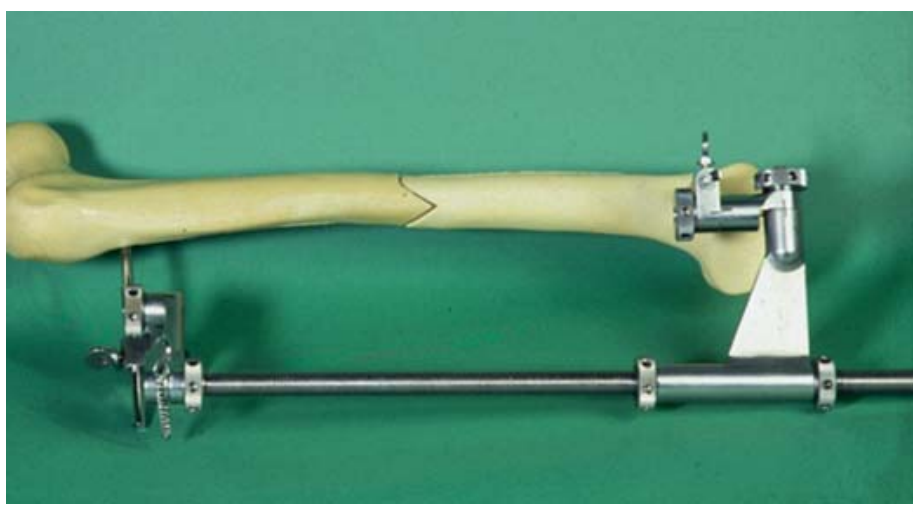

- Abb. 3 AO-Distraktor schematisch am Kunstknochen nach erfolgreicher Reposition der Femurschaftfraktur.

AO-Distraktor in seiner Funktion als passagere Repositionshilfe schematisch dargestellt.

\section{Cerclagen-Osteosynthese}

Eine der grundlegenden Techniken der Frakturreposition und der Stabilisierung von Schaftfrakturen ist die Verwendung einer Cerclage. Hierzu sind verschiedenste Modelle in Verwendung, beginnend von einer einfachen Draht-Cerclage bis hin zu ausgefeilten Band- oder KabelCerclagen, die eine Konnektierung mit winkelstabilen 


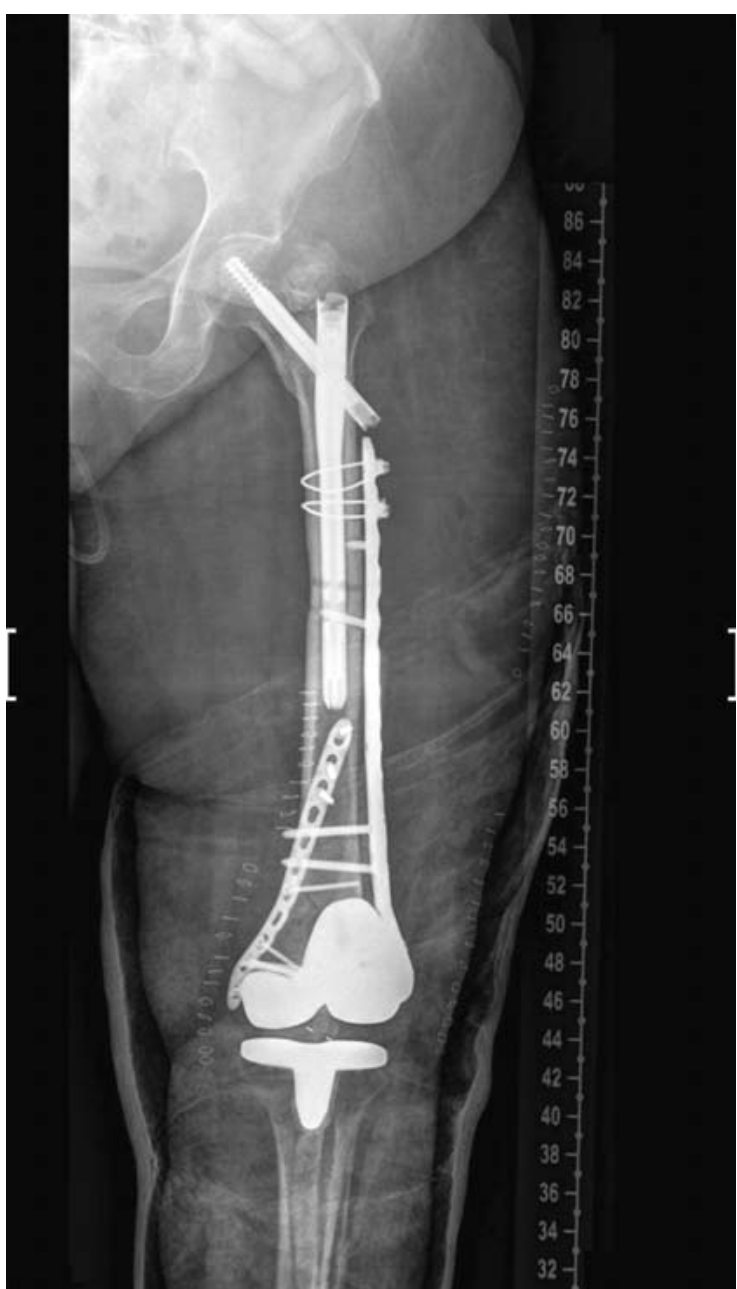

- Abb. 4 Kabel-Cerclage zur Stabilisierung einer Plattenosteosynthese bei einliegendem proximalen Femurnagel. Dabei ist die lediglich monokortikale Verankerungsmöglichkeit von Schrauben (siehe 3. Plattenloch von proximal) und die Konnektierung der Cerclage mit der Platte über spezielle Muffen in den Plattenlöchern zu beachten.

Platten ermöglichen und durch spezielle Spanninstrumente eine biologisch angepasste Vorspannung bereitstellen.

Bei unbestrittenem Nutzen einer Cerclage sollte man sich ein paar Gedanken zu dieser Versorgungstechnik machen.

1. Möchte man die Cerclage als Repositionstool einsetzen?

2. Dient die Cerclage als biomechanischer Stabilisator?

3. Beeinträchtigt die Cerclage die Frakturheilung?

\section{Zu Punkt 1}

In der Versorgung von Schaftfrakturen, insbesondere von Spiral- oder mehrfragmentären Frakturen am Femur, ist die Cerclage ein hervorragendes Hilfsmittel, die einzelnen Fragmente zueinander zu stellen und zu fixieren, um im Anschluss eine intramedulläre Nagelung oder eine

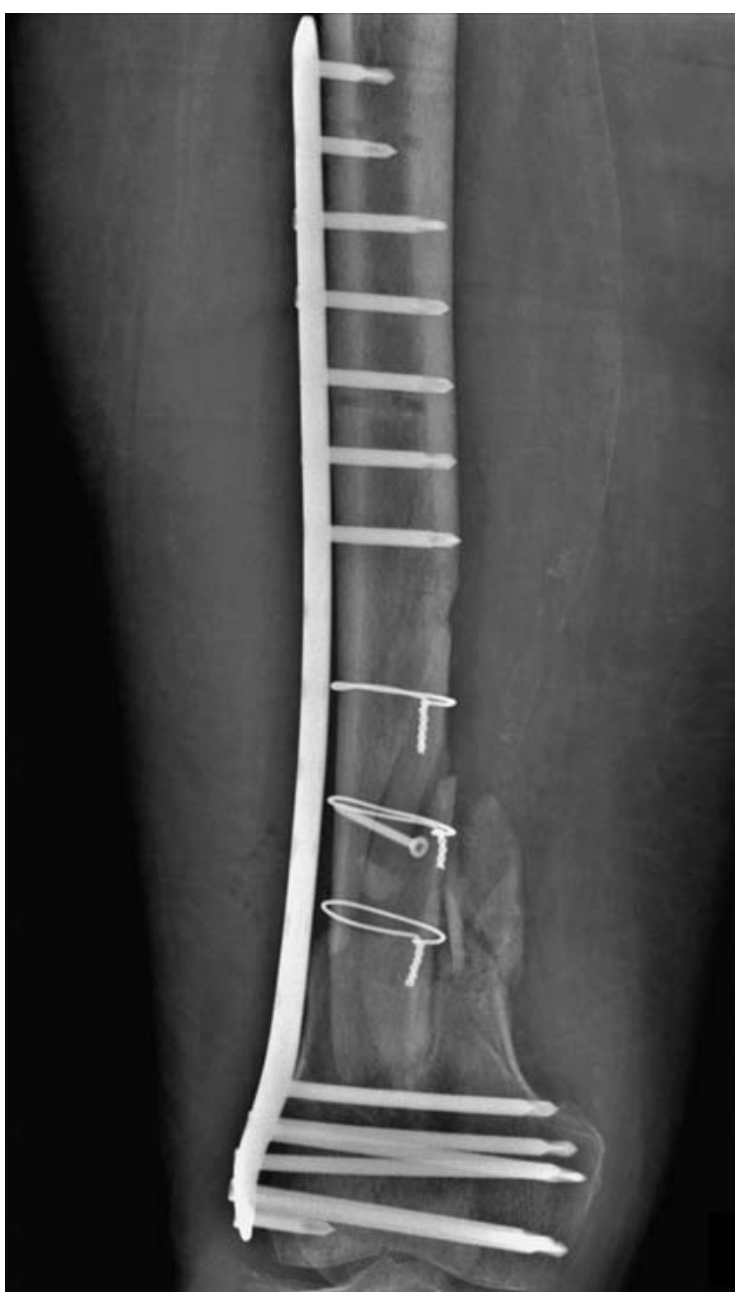

- Abb. 5 Distale Femurfraktur mit Ausbildung einer hypotrophen Pseudarthrose bei mangelnder Biologie nach Osteosynthese mit Cerclagen auf Höhe der Fraktur.

Plattenosteosynthese durchzuführen. Dabei kann über die gewählte Vorspannung der Cerclage eine schrittweise Einpassung der Fragmente vorgenommen werden, z. B. werden bei einer geplanten Nagelosteosynthese ein oder 2 Cerclagen vorgelegt, aber noch nicht komplett geschlossen und nach Einbringung des Nagels die Fragmente bündig um die intramedulläre Schienung geschlossen. Diese Technik braucht etwas Erfahrung, ermöglicht aber sehr gute Ergebnisse.

\section{Zu Punkt 2}

Mithilfe einer Cerclage kann die biomechanische Stabilität einer Osteosynthese deutlich verbessert werden. Dies ist zumeist wünschenswert bei periprothetischen Frakturen unter Erhalt der einliegenden Prothese. Die Kriterien, wann ein Erhalt der Prothese sinnvoll erscheint, hängen in der Hauptsache von der knöchernen Verankerung der Prothese ab; dazu gibt die einschlägige Literatur Algorithmen vor und dies soll hier nicht im Einzelnen abgehandelt 
werden. Unter Umständen hat auch der physiologische Gesamtzustand des Patienten Einfluss auf die Entscheidung z. B. bei schwerkranken bettlägerigen Patienten, für die eine Prothesenwechseloperation ein zu hohes perioperatives Risiko darstellt.

Für den Operateur stellt sich die Frage, ob die Cerclage als Repositionshilfe und gleichzeitig als Stabilisator zum Einsatz kommt. In diesem Fall wird i. d. R. die Cerclage primär gesetzt, belassen und kommt bei einer Plattenosteosynthese unter dem Implantat zu liegen. Oder man wählt die Cerclage als hauptsächlichen Stabilisator und bringt sie über der Platte an. Diese Variante kommt häufig bei periprothetischen Frakturen des proximalen Femurschaftes zum Einsatz und ermöglicht auch die Verwendung von speziellen Konnektoren zur Platte ( $\triangleright$ Abb. 4).

\section{Zu Punkt 3}

Die Cerclagen-Versorgung hat nicht nur o.g. Vorteile, sondern auch zu bedenkende Nachteile. Insbesondere die Biologie sollte nicht missachtet werden. Immer wieder sind Pseudarthrosen zu beobachten, die nach Cerclagen-Versorgungen auftreten. Ein Faktor hierbei ist die Anzahl der Cerclagen. Jede Cerclage stellt eine Strangulation des Periosts dar. Deswegen muss sich der Operateur Gedanken über die Anzahl und die Position der Cerclagen machen. Es gilt abzuwägen: Wie viele Cerclagen sind notwendig, um den erwünschten Effekt in Bezug auf die Stabilität zu erzielen, und ist es nicht im Anschluss an die Osteosynthese möglich, die Anzahl der Cerclagen zu redu-

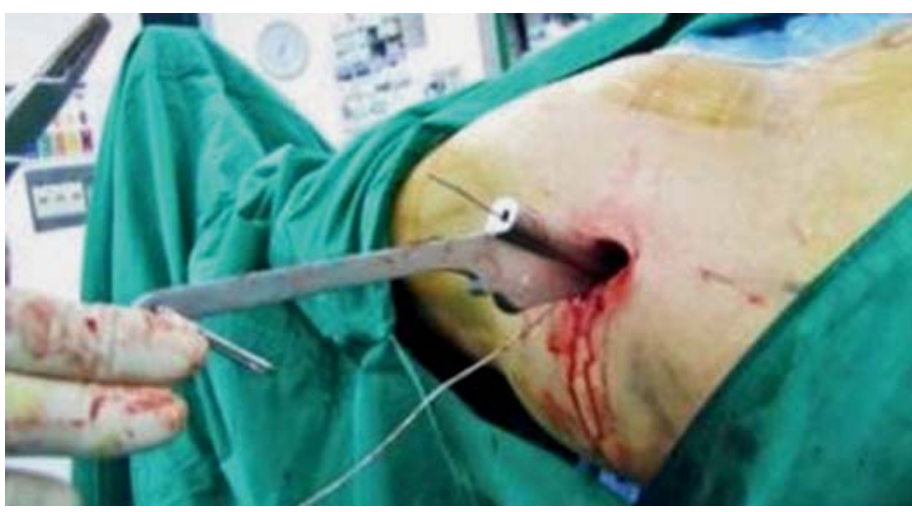

- Abb. 6 Einbringen des „Wire Passer“ über eine kleine und gewebeschonende Schnittinzision.

zieren, um die Biologie nicht nachhaltig zu beeinträchtigen? Auch die Position der Cerclage ist sehr wichtig. Manchmal bedingt die Frakturmorphologie eine Cerclage auf Höhe der Fraktur, um eine gute Reposition zu erhalten. In der Folge zeigt sich aber genau in diesem Bereich eine verzögerte oder ausbleibende Frakturheilung. Auf den Röntgenbildern kann man bildlich den „Würgeeffekt“ der Cerclage nachvollziehen ( $\bullet$ Abb. 5). Deswegen ist zu überdenken, diese primär für die gelungene Reposition wichtige Cerclage am Ende der Operation wieder zu entfernen, da die gewählte Osteosynthese die Aufgabe der Stabilisierung übernimmt.


- Abb. 7 Frakturreposition einer pertrochantären Femurfraktur vor Einbringen des Nagels. a Intraoperatives a.-p. Bild; b axiale Ansicht bei anliegender kollinearer Repositionszwinge. 
Die Wertigkeit einer die Biologie respektierenden OPTechnik ist in der modernen Frakturversorgung unbestritten und ein wesentlicher Bestandteil der operativen Lehre. Dieser Grundsatz sollte auch bei der Verwendung von Cerclagen nicht vernachlässigt werden. Die minimalinvasive und gewebeschonende Vorgehensweise ist bei der Platzierung von Cerclagen mit konventionellen Techniken, wie z.B. Deschamps-Durchzugsinstrument, nur bedingt möglich, insbesondere bei muskel- oder weichteilkräftigen Patienten. Der notwendige Zugang für eine Umschlingung des Schaftes ist u. U. relativ groß und nicht gewebeschonend. Abhilfe schafft ein Instrument, das über eine kleine Inzision um den Knochen geführt werden kann und die Platzierung des Drahtes möglich macht. Der sog. „Wire Passer“ (DePuy-Synthes) stellt ein solches Instrument dar. Über eine ca. $3 \mathrm{~cm}$ lange Inzision kann die 2-schenkelige Zange präzise und knochennahe um den Schaft geführt werden. Dies geschieht erst mit dem einen Schenkel der Zange und dann mit dem anderen, die sich bei exakter Platzierung dann konnektieren und arretieren lassen ( $\bullet$ Abb. 6). Über die Zange, die röhrenförmig ausgelegt ist, lässt sich der Draht problemlos hindurchführen und die Zange, unter Belassen des Drahtes, durch Diskonnektierung entfernen.

Zu beachten bei der Verwendung der Zange ist die streng knochennahe Führung der einzelnen Branchen, damit sich keine wichtigen Weichteilstrukturen zwischen dem Draht und dem Knochen befinden.

Neben dieses für die Biologie nicht zu verachtenden Vorteils des minimalinvasiven Vorgehens erspart der Wire Passer auch einiges an OP-Zeit und Nerven des Operateurs. Es gibt kein umständliches Einfädeln des Drahtes mehr und nachdem auch keine Öse mehr gebogen werden muss, um den Draht in den Deschamp einzuhängen, vermindert sich die Verletzungsgefahr. Dieses Modell einer Cerclage-Zange stellt nur ein Beispiel von unterschiedlichen Lösungen dar, welche die Cerclage-Platzierung erleichtern und einer biologischen Osteosynthese Rechnung tragen.

\section{Kollineare Repositionszwinge}

Initial für die beckenchirurgischen Eingriffe zur Reposition von Azetabulumfrakturen entwickelt, hat sich die kollineare Repositionszwinge in vielen anderen Bereichen einen festen Platz erobert. Durch die weichteilschonende, minimalinvasive Verwendungsmöglichkeit und die Ausübung einer axialen Zugkraft kommt sie als Repositionswerkzeug sowohl bei Schaftfrakturen als auch bei Frakturen mit Gelenkbeteiligung zum Einsatz. Beispielhaft ist in der $>$ Abb. 7 die Versorgung einer pertrochantären Femurfraktur dargestellt. Vor Einbringen des antegraden Femurnagels wird über die Zange ein anatomischer CCD-Winkel hergestellt und damit das postoperative Alignment optimiert.

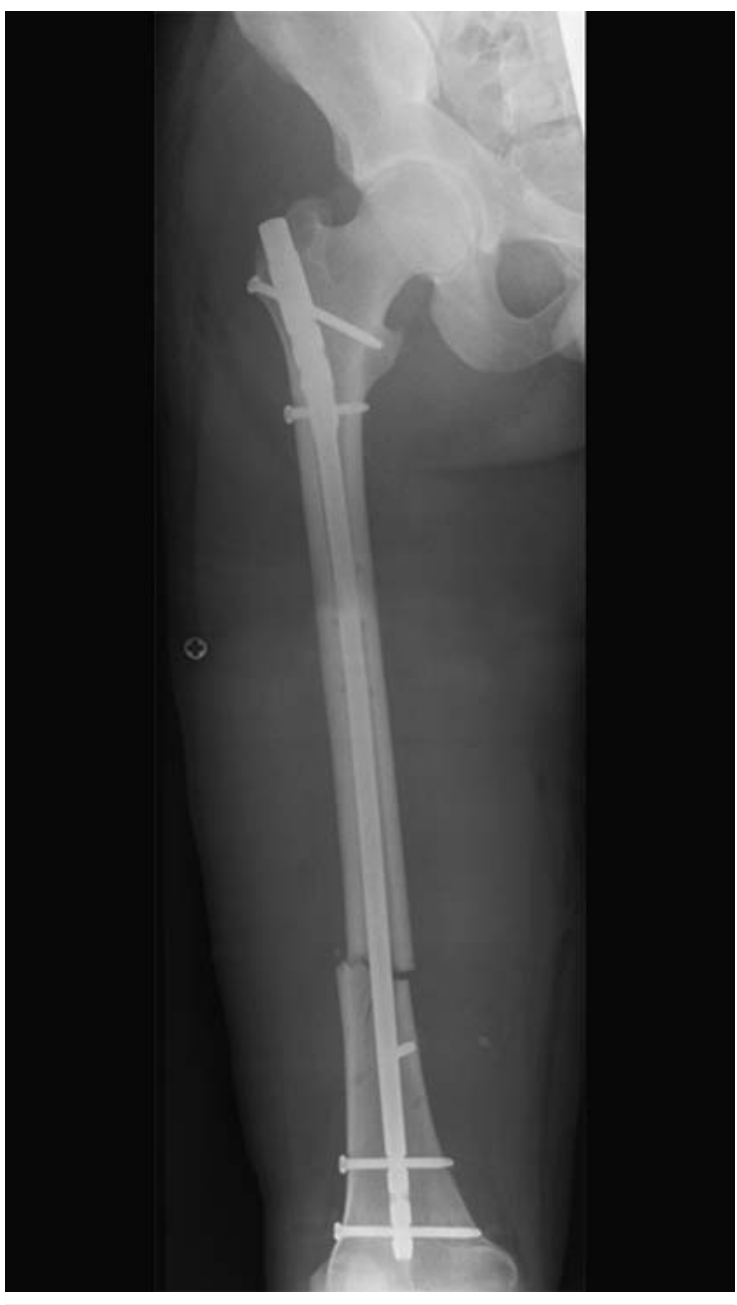

Abb. 8 Durch die Pollerschraube wird eine varische Abweichung des distalen Femurs verhindert.

Merke

Zu beachten:

- Beim Setzen der kollinearen Zwinge keine Verletzung von Gefäßen und Nerven.

- Die punktuelle Kraft der Zwinge ist sehr hoch, somit cave vor iatrogenen Frakturen.

\section{OP-Techniken}

Mit zunehmendem Wissen um die biologischen Zusammenhänge der Frakturheilung und durch neue Implantate sind OP-Techniken in einem stetigen Wandel. Mit der Einführung von intramedullären Kraftträgern, wie z.B. Pivot-Nägeln oder Schaftnägeln jeglicher Art, hat auch das Verständnis um die intraoperative Schonung der umgebenden Weichteile und die sich daraus ergebende verbesserte Frakturheilung zugenommen. Dieses Prinzip der indirekten Reposition mit weichteilschonender, frakturferner Einbringung des Osteosynthesematerials ist heute auch bei Plattenosteosynthesen Standard. Gerade aus dem Umstand der indirekten Frakturreposition bedarf es 


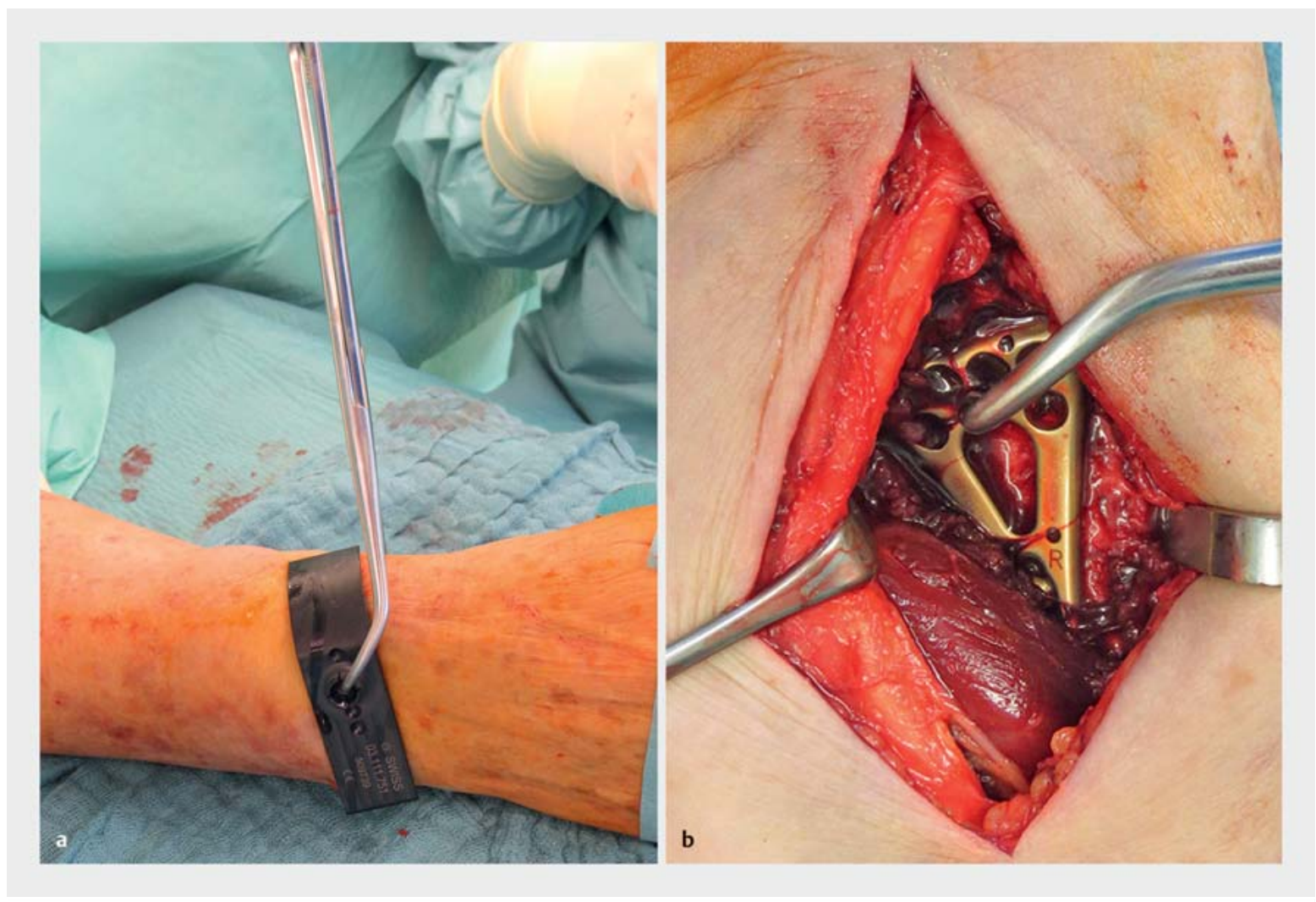

- Abb. 9 a, b Versorgung einer distalen Radiusfraktur über volaren Zugang unter Zuhilfenahme der J-Repositionszange.

manches operativen Tricks, um dennoch ein gutes Repositionsergebnis zu erhalten. Klassische OP-Techniken sollten auch nicht in Vergessenheit geraten, da sie manchmal hilfreich bei komplikativen Verläufen sein können oder eine Ergänzung in Hinblick auf belastungsstabile Osteosynthesen bei osteoporotischen Frakturen in der Alterstraumatologie darstellen.

\section{Pollerschraube}

Die intramedulläre Nagelung hat einen Siegeszug in der modernen Frakturversorgung vollzogen. Von der einfachen intramedullären Schienung langer Röhrenknochen kam es durch zunehmende Verbesserung der OPTechnik und der Implantate zu einer Ausdehnung der Indikation auch bei intraartikulär auslaufenden Schaftfrakturen. Um jedoch eine achsgerechte Ausrichtung des Röhrenknochens zu erreichen, bedarf es manchmal der Verwendung von Hilfsmitteln. Neben Draht-Cerclagen können auch sog. „Pollerschrauben“ verwendet werden. Diese erfüllen neben der Ausrichtung der Fragmente zum Nagel und damit in die anatomische Achse zusätzlich die Funktion der Stabilitätserhöhung. Wichtig ist für den/die Operateur/-in, sich schematisch mit der Wirkungsweise einer Pollerschraube auseinanderzusetzen und sich daraus die optimale Lokalisation der Schraube zu erarbeiten [2]. In der $\triangleright$ Abb. 8 ist die Verwendung einer Pollerschraube und ihre Wirkung an einem Beispiel dargestellt.

\section{J-Repositionszange distaler Radius}

In der Versorgung von distalen Radiusfrakturen hat sich in den letzten Jahren zunehmend eine Versorgung mittels volarer winkelstabiler Platten durchgesetzt $[3,4]$. Mit dieser OP-Technik sind gute Repositionsergebnisse und eine frühfunktionelle Nachbehandlung zu erreichen. Eine Limitation der OP-Technik ist bei intraartikulären Impressionen und bei einem dorsalen Kantenfragment gegeben. Diese Frakturen bedürfen häufig eines dorsalen Zugangs, der operativ anspruchsvoller ist und mit einer höheren Komplikationsrate einhergeht. Eine Erweiterung der OP-Technik des volaren Zugangs stellt die Kombination mit der J-Repositionszange dar. Über den klassischen volaren Zugang wird die jeweilige distale Radiusplatte am Radiusschaft positioniert. Im nächsten Schritt erfolgt die Reposition des dorsalen Kantenfragments über die J-Zange mit der Platte als Widerlager, wie in $\mathbf{A} \mathbf{A b} \mathbf{b} . \mathbf{9} \mathbf{a}$ und $\mathbf{b}$ dargestellt. Darüber sind in vielen Fällen gute anatomische Repositionsergebnisse zu erreichen und ein dorsaler Zugang ist vermeidbar. Geeignet sind nur Frakturen, die nicht einer Einsichtnahme in das Gelenk bedürfen.

Merke

Zu beachten:

- korrekte Plattenpositionierung volar

- Bildwandlerkontrolle der korrekten Zangenposition

- kein Druckschaden der Weichteile insbesondere der Haut 


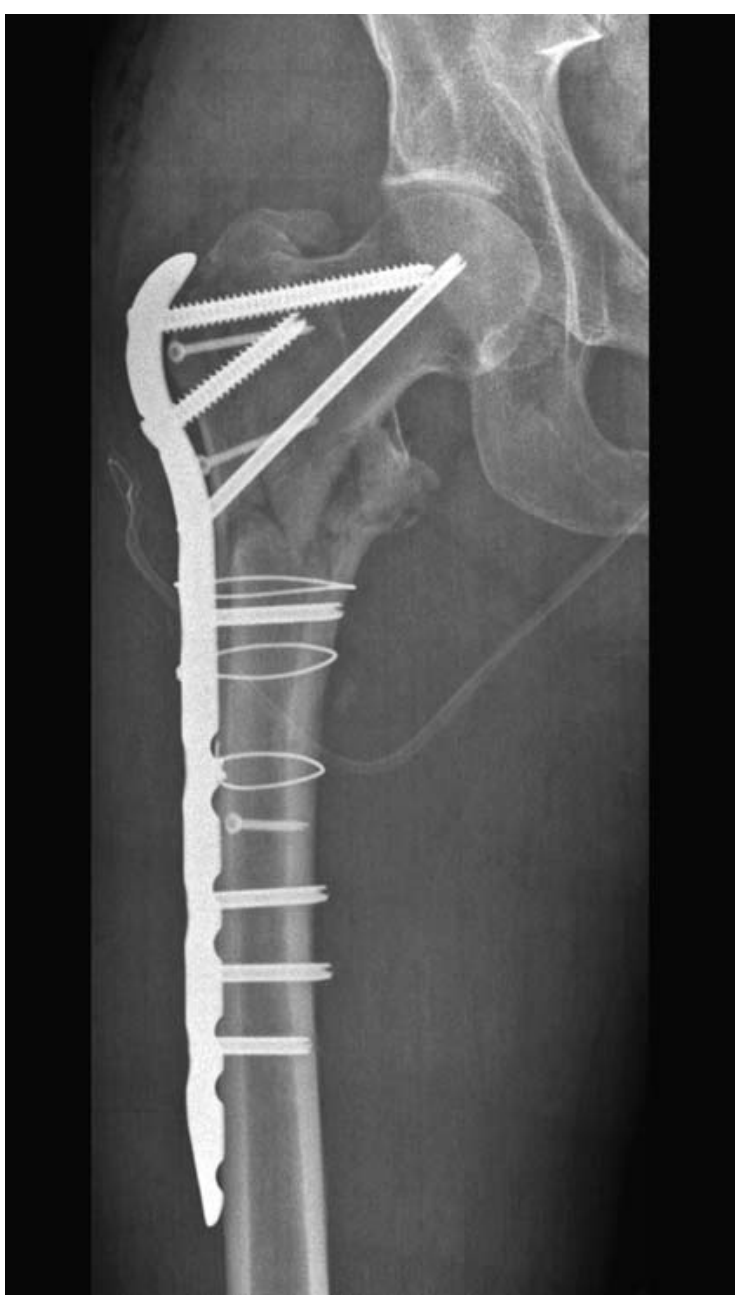

- Abb. 10 Intertrochantäre Femurfraktur mit LC-Platte versorgt.

\section{Klingenplatte}

Die Standard-OP-Technik in der Versorgung von intertrochantären Femurfrakturen ist heutzutage die intramedulläre Schienung mittels eines antegraden, langen Femurnagels mit der Option einer Verriegelung im Schenkelhals. Bei komplexen Frakturen ist eine geschlossene Reposition über eine Extension nicht immer möglich, sodass ein limitierter Zugang zur direkten Reposition der Fragmente mit Draht-Cerclagen und dem Nagel kombiniert wird. Eine mögliche Alternative stellen neuere winkelstabile Platten dar, wie in der $\boldsymbol{A} \mathbf{A b b} \mathbf{1 0}$ dargestellt.

Sowohl die Nagelosteosynthese als auch die Plattenosteosynthese führen nicht immer zum gewünschten Heilungsergebnis und es kommt zu Pseudarthrosenbildung und Implantatversagen. Im dargestellten Fall ist die Reposition des CCD-Winkels nicht beachtet worden. Es kam zu einer Beinlängenverkürzung, einer Überlastung des Implantats und zur Ausbildung einer Pseudarthrose.

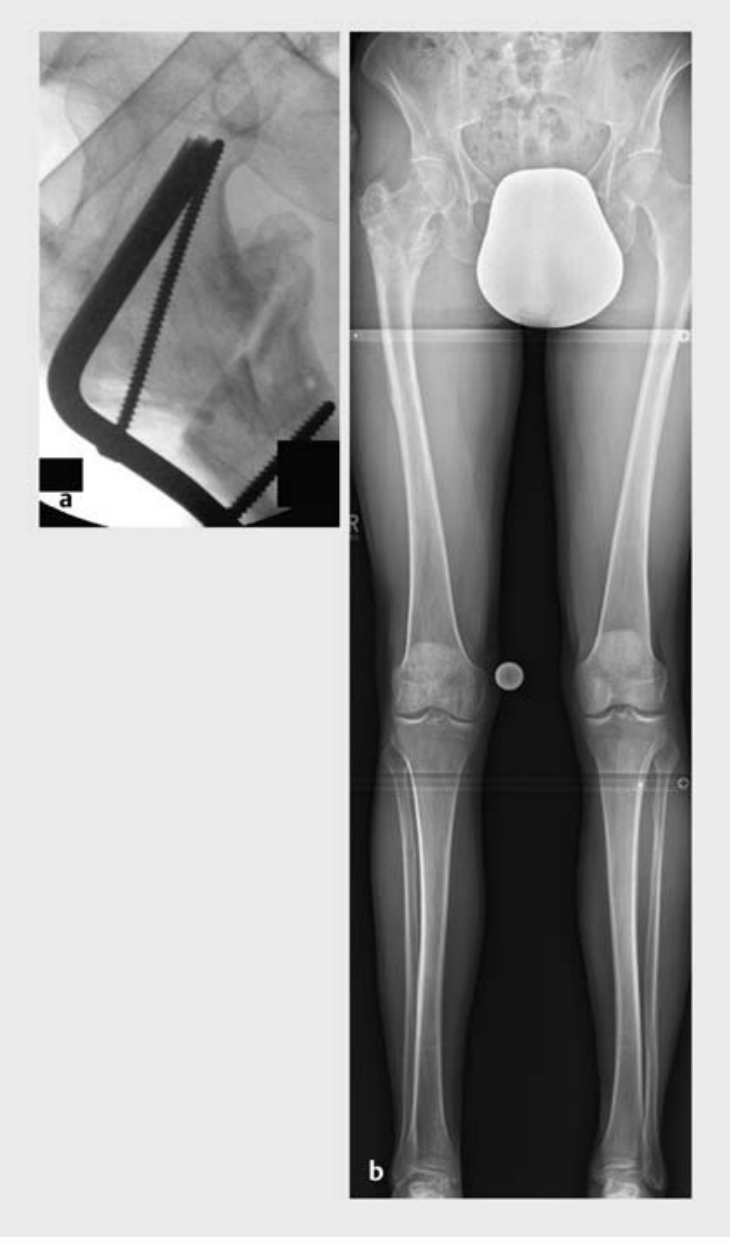

- Abb. 11 a Umstellungsosteotomie mit abschließendem CCD-Winkel von $140^{\circ}$ und Stabilisierung mit 7-LochKlingenplatte. Dabei Osteotomiekompression über den Plattenspanner. Quelle: BG Klinik Tübingen. b Ausheilung nach 15 Monaten nach Plattenentfernung bei ausgeglichener Beinlänge.

Zur Rettung der Situation mit Wiederherstellung der anatomischen Winkel und Ausheilung der Fraktur bedarf es einer Umstellungsosteotomie auf Höhe der Pseudarthrose und eines winkelstabilen, belastbaren Implantats [5]. Ein mögliches Verfahren ist der Wechsel auf einen proximalen, intramedullären Nagel. Dabei ist aber zu beachten, dass der Eintrittspunkt der Schenkelhalsverriegelungsschraube oder -klinge nicht auf der Höhe der Osteotomie ist und dass im Schenkelhals und Femurkopf, bedingt durch die vorherigen Implantate, genug knöcherne Substanz zur sicheren Verankerung vorhanden ist. Eine Alternative stellt die klassische Winkel- oder Klingenplatte dar. Mit der Klingenplatte ist es möglich, im Bereich des Schenkelhalses eine gute Verankerungsposition zu wählen und über ein Plattenspanngerät die Osteotomie unter Kompression zu setzen ( $\boldsymbol{A}$ Abb. 11 a). Die OP-Technik ist heutzutage nicht mehr geläufig und bedarf eines mit dem Verfahren vertrauten Operateurs. Durch die hohe 


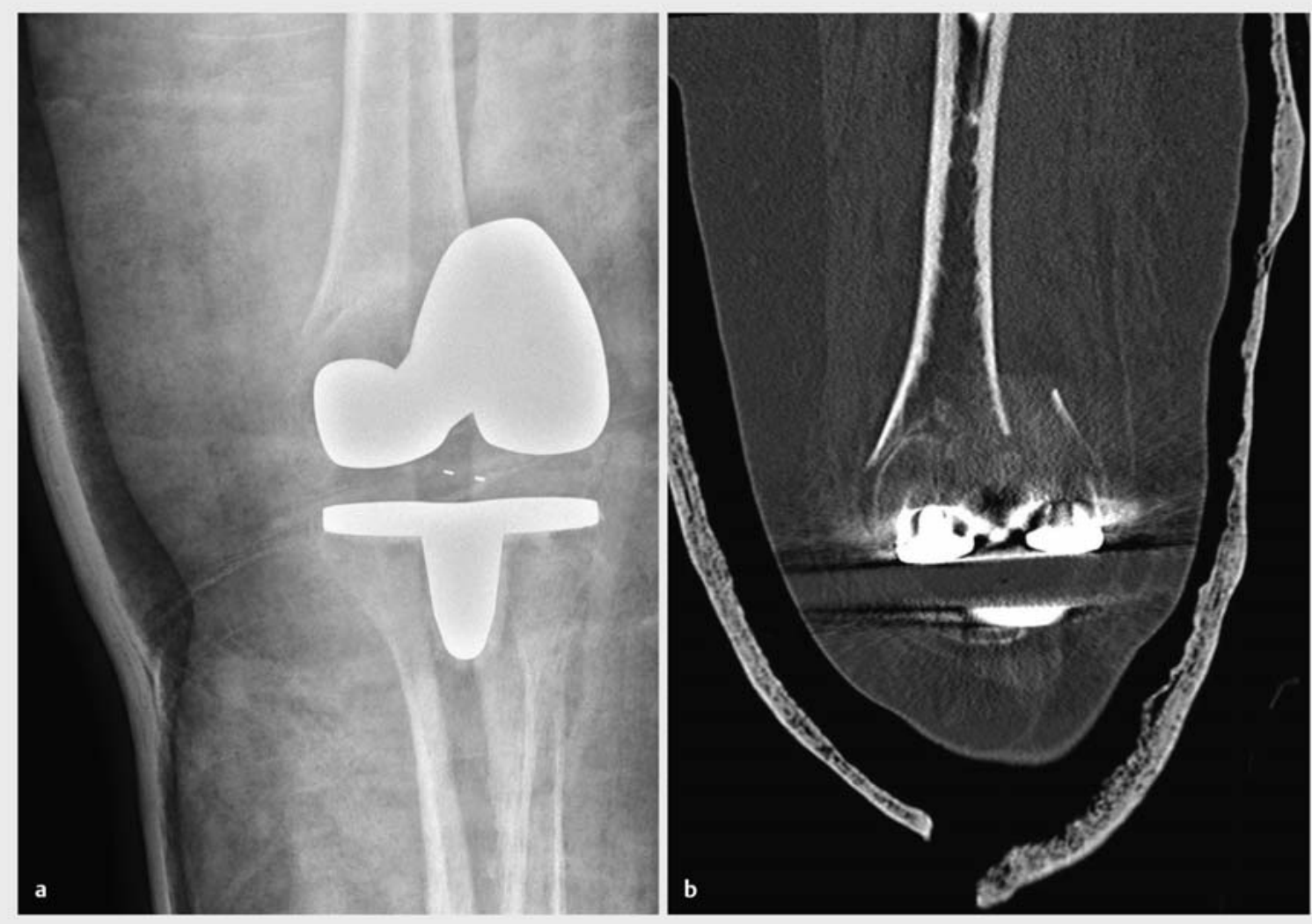

- Abb. 12 a Interprothetische distale Femurfraktur bei proximalem Femurnagel und Knieendoprothese. b Knieprothesen ohne Lockerungszeichen (Lewis-Rorabeck Typ II).

Stabilität der Osteosynthese mit Kompression des Frakturspaltes sind i.d. R. die Frakturheilung zu erreichen und gute Ausheilungsergebnisse zu erzielen ( $\bullet$ Abb. 11 b).

\section{Doppelplattenosteosynthese des distalen Femurs}

Einhergehend mit der demografischen Entwicklung der Bevölkerung ist auch eine deutliche Zunahme von Frakturen bei minderer Knochenqualität zu verzeichnen. Eine besondere Herausforderung stellen Frakturen der unteren Extremitäten dar, da postoperativ eine Teilbelastung aufgrund der physischen und psychischen Möglichkeiten des Patienten nicht immer möglich ist. Anzustreben ist eine OP-Technik, die eine Vollmobilisation des Patienten weitgehend ermöglicht. Im Bereich des proximalen Femurs ist dies zumeist unter Verwendung von intramedullären Verfahren gut möglich, aber im Bereich des distalen Femurs, insbesondere bei einliegender Knieendoprothese, gibt es Limitationen, wenn kniegelenksnahe nur ein kurzer metaphysärer Knochenblock besteht, ein Prothesenwechsel bei festsitzenden femoralen und tibialen Komponenten nicht indiziert ist (Lewis-Rorabeck Typ I und II).
Nicht immer stellt die retrograde Nagelosteosynthese ein geeignetes Verfahren dar, da zum einen die femorale Komponente ein Einbringen des Nagels ermöglichen muss, d. h. der Raum zwischen dem medialen und lateralen Oberflächenersatz der Femurkondylen muss groß genug sein, um den Nagel intramedullär platzieren zu können; zum anderen finden sich gehäuft Patienten, die zusätzlich einen proximalen Femurnagel oder eine Hüftendoprothese haben ( $\vee$ Abb. 12a und 12b).

In diesen Fällen ist die diaphysäre Brücke zwischen den Implantaten zu kurz $(<4 \mathrm{~cm})$ und eine mögliche Sollbruchstelle. Ein alternatives Verfahren ist eine sog. Doppelplattenosteosynthese des distalen Femurs [6]. Dabei wird eine laterale winkelstabile Großfragmentplatte mit einer anterior-medialen torquierten Kleinfragment-LCP kombiniert. Die operativen Schritte sind wie folgt durchzuführen:

In einem 1. Schritt wird über einen ca. $6 \mathrm{~cm}$ langen Schnitt über der lateralen Femurkondyle und entsprechender Weichteilpräparation eine ausreichend lange winkelstabile Platte unter Zuhilfenahme eines Zielbügels eingeschoben. Dabei ist auf 2 Punkte besonders zu ach- 


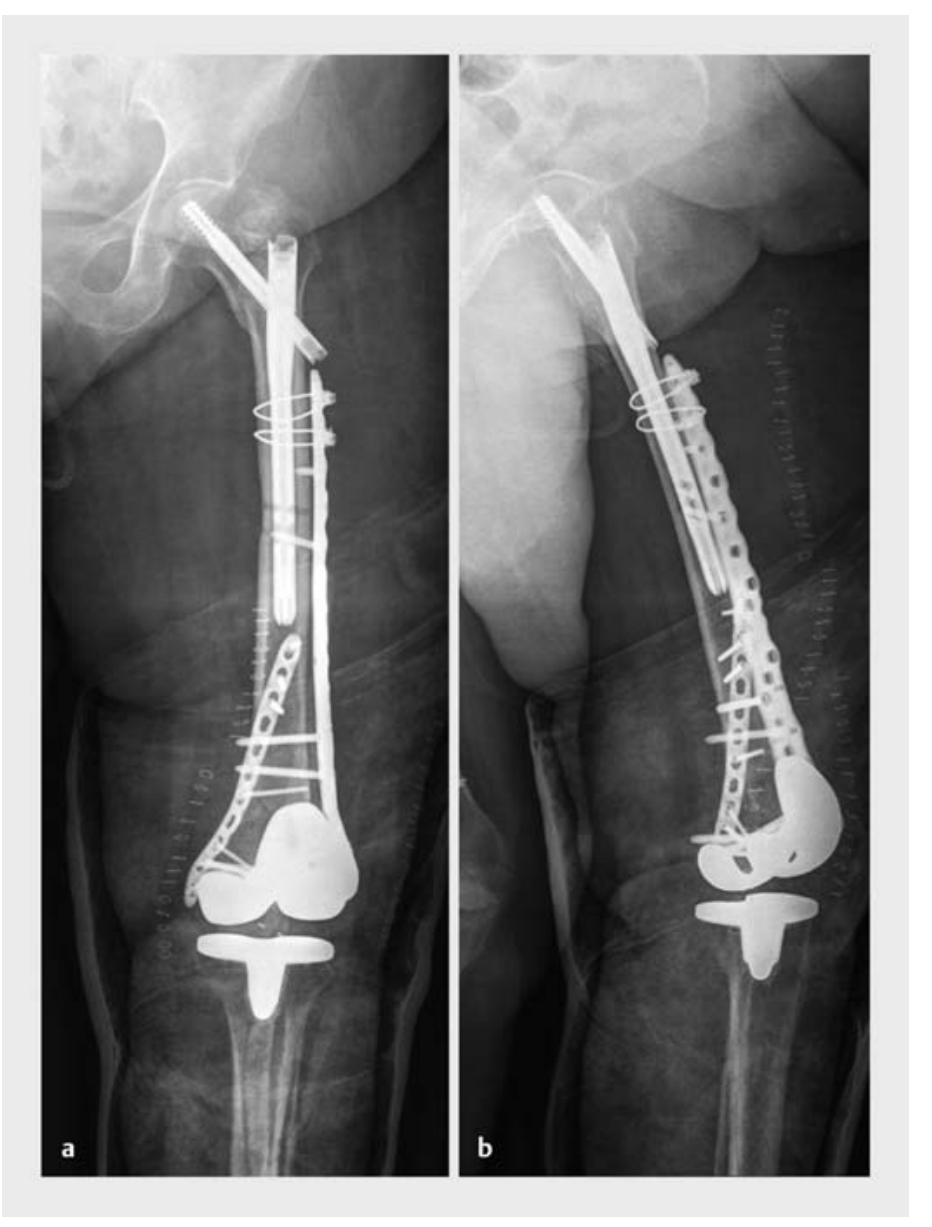

- Abb. 13 a, b Röntgen a.-p. und seitlich, belastungsstabile Doppelplattenosteosynthese.

ten: 1. Die Länge der Platte sollte bei proximalem Implantat die distalen Anteile des Implantats deutlich überbrücken, um eine Stressfraktur zu vermeiden. 2. Man muss auf eine achsgerechte Reposition auch nach Fixierung der Platte Wert legen. Nach primärer Fixierung der Fraktur mittels lateraler Plattenosteosynthese wird in einem 2. OP-Schritt eine zusätzliche anterior-mediale Stabilisierung vorgenommen. Da bisher keine vorkonfigurierten Implantate erhältlich sind, eignet sich eine 12-Loch-3,5mm-LCP, die entsprechend der gegebenen anatomischen Verhältnisse vorgeformt wird. Um eine ausreichende Fixierung über dem medialen Kondylus zu erreichen, muss die Platte im distalen Anteil dem Kondylus angeformt werden ( $\vee$ Abb. 13a und $\mathbf{1 3}$ b). Eine streng mediale Platzierung der Platte birgt ein hohes Risiko auf eine Verletzung der Gefäße, somit ist dies zu vermeiden und die Platte im mittleren und proximalen Anteil um ca. $45^{\circ} \mathrm{zu}$ torquieren, sodass sie ventromedial auf der Femurdiaphyse zu liegen kommt (siehe $\mathbf{A b b}$. 10). Operativ wird ein ca. $10 \mathrm{~cm}$ langer Schnitt über dem anterior-medialen Oberschenkel angelegt und die Weichteile über dem medialen Kondylus präpariert. Die Muskulatur wird auf dem
Periost untertunnelt nach anterior-proximal und die vorgeformte Platte eingeschoben. Die Fixierung erfolgt im distalen Anteil mit zumindest 3 winkelstabilen Schrauben und proximal ebenfalls mit 3 bikortikalen winkelstabilen Schrauben über Zielbuchsen von ventral.

Diese kombinierte Doppelplattenosteosynthese, wie in - Abb. 10 dargestellt, hat eine hohe Primärstabilität und ermöglicht zumeist eine frühzeitige Vollbelastung trotz osteoporotischer Knochenverhältnisse.

Merke

Zu beachten:

- ausreichende Überlappung der Osteosynthese und des intramedullären Implantats

- korrekte anatomische Beinachse

- ausreichende Schraubenanzahl im distalen Fragment

- anteriore Plattenlage im proximalen Anteil der medialen Osteosynthese

- keine monokortikale Verschraubung

\section{Fazit}

Die Kombination aus aktuellen OP-Techniken und dem Wissen um klassische Verfahren und Implantate ist bei der Behandlung von komplexen Frakturen von unschätzbarem Wert. Ohne Frage können wir heutzutage auf anatomisch optimierte und nach dem aktuellen Stand der Wissenschaft gefertigte Implantate zurückgreifen, aber der Erfolg der Frakturversorgung hängt immer von der Qualität der Reposition ab. Die eingehende Beschäftigung mit klassischen Repositions- und OP-Techniken ist nicht nur spannend, sondern in schwierigen Situationen sehr hilfreich und führt zu besseren Ergebnissen.

\section{Autorinnen/Autoren}

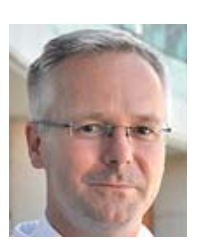

\section{Thomas Freude}

MD, Univ.-Prof., Vorstand Universitätsklinik für Orthopädie und Traumatologie, Paracelsus Medizinische Privatuniversität Salzburg

\section{Korrespondenzadresse}

\section{Thomas Freude, MD}

Universitätsklinik für Orthopädie und Traumatologie Paracelsus Medizinische Privatuniversität (PMU)

Müllner Hauptstr. 48

5020 Salzburg

Österreich

Tel.: + 43/57255-50001

Fax: $+43 / 57255-50004$

t.freude@salk.at 


\section{Interessenkonflikt}

Der Autor erklärt, dass kein Interessenkonflikt vorliegt.

Literatur

[1] Perren SM, Perren T, Schneider E. [Are the terms „biology“ and „osteosynthesis“ contradictory?]. Ther Umsch 2003; 60: 713721

[2] Hannah A, Aboelmagd T, Yip G et al. A novel technique for accurate Poller (blocking) screw placement. Injury 2014; 45: 1011-1014

[3] Loisel F, Kielwasser H, Faivre G et al. Treatment of distal radius fractures with locking plates: an update. Eur J Orthop Surg Traumatol 2018. doi:10.1007/s00590-018-2274-z
[4] Madsen ML, Wæver D, Borris LC et al. Volar plating of distal radius fractures does not restore the anatomy. Dan Med J 2018; 65: pii: A5497

[5] Hartline BE, Achor TS. Use of the 95-degree angled blade plate to treat a proximal femur fracture. J Orthop Trauma 2018; 32 (Suppl. 1): S26-S27

[6] Lenz M, Perren SM, Gueorguiev B et al. Mechanical behavior of fixation components for periprosthetic fracture surgery. Clin Biomech (Bristol, Avon) 2013; 28: 988-993

Bibliografie

DOI https://doi.org/10.1055/a-0725-4367

Online-publiziert 18.12.2018 | OP-JOURNAL 2019; 35: 28-37

(c) Georg Thieme Verlag KG Stuttgart · New York ISSN 0178-1715 